\title{
Journal of

\section{Mechanism of Si intercalation in defective graphene on SiC}

\author{
T. P. Kaloni, M. Upadhyay Kahaly, Y. C. Cheng and U. Schwingenschlögl* \\ Received 1st August 2012, Accepted 1st October 2012 \\ DOI: $10.1039 / c 2 j m 35127 \mathrm{~g}$
}

Previously reported experimental findings on Si-intercalated graphene on $\mathrm{SiC}(0001)$ seem to indicate the possibility of an intercalation process based on the migration of the intercalant through atomic defects in the graphene sheet. We employ density functional theory to show that such a process is in fact feasible and obtain insight into its details. By means of total energy and nudged elastic band calculations we are able to establish the mechanism on an atomic level and to determine the driving forces involved in the different steps of the intercalation process through atomic defects.

\section{Introduction}

One of the possible methods for the mass-scale production of graphene is thermal decomposition of $\mathrm{SiC} .{ }^{1}$ In this process, $\mathrm{Si}-\mathrm{C}$ bonds are broken and the detached $\mathrm{C}$ atoms form graphene, under annealing at a sufficiently high temperature. This growth technique does not require transfer of graphene onto another substrate, which prominently improves the film quality ${ }^{2}$ and clearly is a huge advantage for electronic applications. It has been reported in ref. 3 and 4 that a buffer layer forms between the graphene and $\mathrm{SiC}$ substrate, which suppresses the substrate effect on the graphene as it passivates the dangling bonds of the substrate. ${ }^{5,6}$ Formation of quasi freestanding graphene is then possible by $\mathrm{H}$ and $\mathrm{F}$ intercalation between the buffer layer and substrate., ${ }^{7,8}$

In the last few years, huge efforts have been undertaken to understand the electronic, structural, and magnetic properties of intercalated graphene systems both with and without the substrate. ${ }^{9-13}$ Intercalation of $\mathrm{Si}$ is particularly interesting due to the fact that this material forms the basis of modern electronics technology. In addition, $\mathrm{Si}$ intercalated graphene systems are characterized by a particularly high stability. Recently, Si intercalated graphene on C/Si-terminated SiC has been reported experimentally. ${ }^{14,15}$ In ref. 14 the authors have employed low-energy electron microscopy to study the surface morphology during the growth of graphene and the Si intercalation process. The dependence of the intercalation on the annealing temperature has been addressed. Surprisingly, the authors find that $\mathrm{Si}$ atoms are rather gentle to the $\mathrm{Clayers}$ and do not create any type of defect or dislocation even up to a temperature of $1000{ }^{\circ} \mathrm{C}$. However, the success of $\mathrm{Si}$ intercalation seems to depend on the presence of defects in the graphene sheet. The authors observe that $\mathrm{Si}$ intercalation is not possible in defect-free graphene, especially that

PSE Division, KAUST, Thuwal 23955-6900, Kingdom of Saudi Arabia. E-mail: udo.schwingenschlogl@kaust.edu.sa; Tel: +966 (0) 544700080 grown ex situ. Existing defects or domain boundaries appear to play a key role. In addition, at a high temperature of about $1400 \mathrm{~K}$ deintercalation of $\mathrm{Si}$ and formation of $\mathrm{SiC}$ on the surface are observed. The importance of defects for the intercalation may distinguish $\mathrm{Si}$ from other intercalants such as $\mathrm{H}, \mathrm{Li}, \mathrm{F}, \mathrm{K}$, and Ge. .,8,16-19 $^{-19}$

A deeper insight into the mechanism of $\mathrm{Si}$ intercalation through atomic defects is missing so far. In particular, the energy costs associated with the different steps of the intercalation process as well as the structural and electronic properties of the system during the process are of great interest for the design of electronic devices. In the present work we therefore use first principles calculations based on density functional theory to investigate the mechanism underlying the $\mathrm{Si}$ intercalation of graphene on $\mathrm{SiC}(0001)$.

\section{Methodology}

The Quantum-ESPRESSO code $^{20}$ is employed in our study, using the Perdew-Burke-Ernzerhof parametrization of the generalized gradient approximation for the exchange-correlation potential with a plane wave cutoff energy of $816 \mathrm{eV}$. Structure relaxation is performed up to a force convergence of $0.05 \mathrm{eV} \AA^{-1}$. Self-consistency of the charge distribution is assumed for an energy convergence of $10^{-5} \mathrm{eV}$. Our supercell contains a $4 \times 4 \times 1$ graphene sheet with a single monovacancy (31 $\mathrm{C}$ atoms) on top of a $\mathrm{C}$ buffer layer and a $2 \sqrt{3} \times 2 \sqrt{3} R 30^{\circ} \mathrm{SiC}(0001)$ substrate. A $15 \AA$ thick vacuum layer prevents artificial interaction due to the periodic boundary conditions perpendicular to the graphene sheet. The Si-terminated $\mathrm{SiC}(0001)$ substrate is modeled by a slab of $4 \mathrm{SiC}$ layers, where the dangling bonds at the bottom of the slab are saturated by $\mathrm{H}$ atoms. The top $\mathrm{Si}$ atoms of the substrate interact with the $\mathrm{C}$ buffer layer, which results in a prominent buckling. An automatically generated Monkhorst-pack $4 \times 4 \times 1 \mathrm{k}$-mesh is used. The van der Waals interaction is taken into account for all the systems under study to obtain accurate distances between the atomic layers. ${ }^{21,22}$ Energy barriers are calculated using the climbing image nudged elastic band method. ${ }^{23}$

We report in the following only the energetically most favorable configurations out of the many possible configurations which we have investigated. These configurations are depicted in Fig. 1, all being fully relaxed. A defective graphene sheet lies on top of the $\mathrm{C}$ buffer layer with a $\mathrm{Si}$ atom far away from the graphene sheet (configuration 1A) and trapped at the defect (configuration 1B). Configurations $2 \mathrm{~A}$ and $2 \mathrm{~B}$ describe the same situations for a second $\mathrm{Si}$ atom and configurations $3 \mathrm{~A}$ and $3 \mathrm{~B}$ for a third $\mathrm{Si}$ atom. 
$1 \mathrm{~A}$

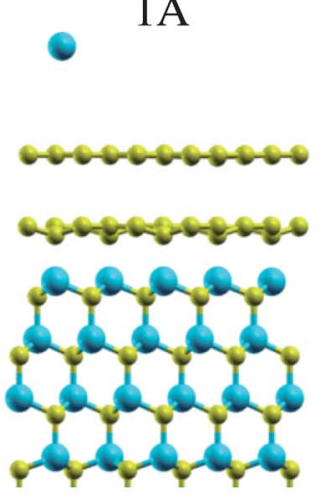

$2 \mathrm{~A}$

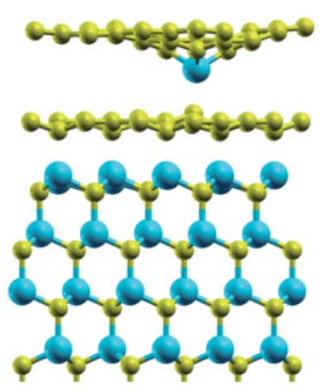

$3 \mathrm{~A}$

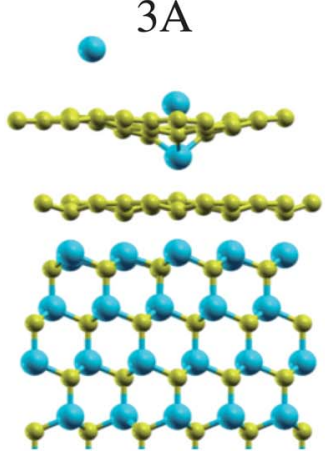

1B

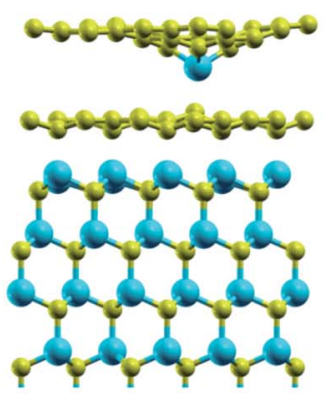

$2 \mathrm{~B}$

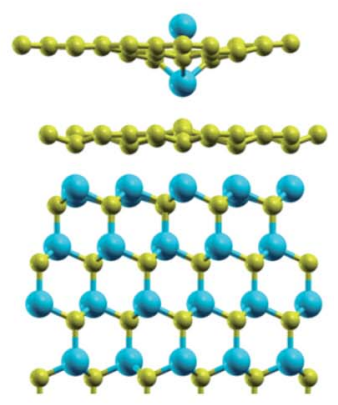

3B

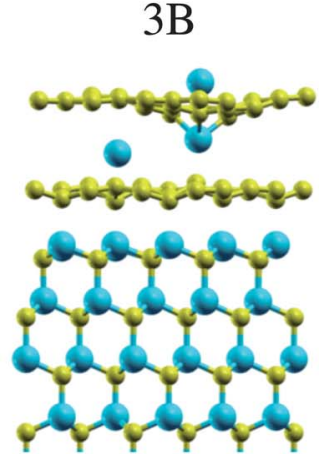

Fig. 1 Side views of the configurations addressed in the present study. Blue (big) and yellow (small) circles represent the $\mathrm{Si}$ and $\mathrm{C}$ atoms, respectively. Configurations $1 \mathrm{~A} / 1 \mathrm{~B}$ refer to the first approaching $\mathrm{Si}$ atom, configurations $2 \mathrm{~A} / 2 \mathrm{~B}$ to the second, and configurations $3 \mathrm{~A} / 3 \mathrm{~B}$ to the third. The buckled $\mathrm{C}$ layer on top of the $\mathrm{SiC}(0001)$ substrate is the buffer layer.

Comparing the energetics of these six configurations in the following will allow us to understand the mechanism of the Si intercalation and the structural modifications which the system undergoes during this process.

\section{Structural analysis}

In configuration $1 \mathrm{~A}$ the average $\mathrm{Si}-\mathrm{C}$ bond length is found to be $1.96 \AA$, i.e., $3.6 \%$ longer than the bulk $\mathrm{SiC}$ bond length of $1.89 \AA^{24}$ The top $\mathrm{Si}$ atoms form covalent bonds with the $\mathrm{C}$ buffer layer above, resulting in a prominent buckling of the latter with an amplitude of almost $0.4 \AA$. Since there is little interaction with the $\mathrm{C}$ buffer layer, the defective graphene sheet shows essentially no buckling. The average distance to the $\mathrm{C}$ buffer layer is found to be $3.3 \AA$, which agrees well with the interlayer distance in graphite.

The $\mathrm{Si}$ atom approaches the defect in the graphene sheet and finds there an energetically stable state (above the sheet). A similar stable state exists symmetrically below the sheet, see configuration 1B in Fig. 1. A nudged elastic band calculation shows that between these two states an energy barrier of $1.99 \mathrm{eV}$ has to be overcome. The value of the barrier suggests that $\mathrm{Si}$ intercalation is not possible at low or room temperature but that a substantially high temperature is required, consistent with the experimental situation. The total energy of configuration $1 \mathrm{~B}$ is $11.02 \mathrm{eV}$ lower than that of configuration 1A. Table 1 summarizes the energetics of the configurations shown in Fig. 1. While the $\mathrm{C}$ buffer layer and substrate virtually do not react to the adsorption, the $\mathrm{Si}$ atom forms bonds with three neighbouring $\mathrm{C}$ atoms. This saturation of the dangling $\mathrm{C}$ bonds at the defect explains the huge energy gain of $11.02 \mathrm{eV}$ and results in a significant distortion and buckling of the defective graphene sheet. The $\mathrm{C}-\mathrm{C}$ bond lengths around the defect vary between 1.39 and $1.40 \AA$, while the bond length in pristine graphene is $1.42 \AA$. The $\mathrm{C}-$ Si bond lengths are found to be close to $1.79 \AA$. A reduction of the in-plane bond angles after the $\mathrm{Si}$ adsorption and the three-dimensional distortion of the graphene sheet indicate that the $\mathrm{sp}^{2}$ hybridization of the ideal honeycomb lattice is replaced by a mixture of $\mathrm{sp}^{2}$ and $\mathrm{sp}^{3}$ states.

From configurations $1 \mathrm{~A}$ and $1 \mathrm{~B}$ we have seen that a single $\mathrm{Si}$ atom which approaches a defect in the graphene sheet by chance will be adsorbed. However, the energetics show that it cannot migrate through the defect to become intercalated. We therefore will next address the effects of additional $\mathrm{Si}$ atoms, see configuration $2 \mathrm{~A}$. If a second $\mathrm{Si}$ atom by chance comes close to the defect, which is already occupied by one $\mathrm{Si}$ atom, it will also be adsorbed. Our calculations clearly show that there exists a second stable adsorption site for a $\mathrm{Si}$ atom slightly on top of the graphene sheet, see configuration $2 \mathrm{~B}$ in Fig. 1. Since the second $\mathrm{Si}$ atom in the relaxed structure is located only $0.80 \AA$ above the defect, the initially adsorbed $\mathrm{Si}$ atom is pushed downwards. However, there remains a local energy minimum in which this atom is trapped. As the total energy is concerned, configuration $2 \mathrm{~B}$ is found to be $2.45 \mathrm{eV}$ lower than configuration $2 \mathrm{~A}$. This is the energy gain due to the adsorption of the second $\mathrm{Si}$ atom. The structures of the $\mathrm{C}$ buffer layer and the substrate are little affected by the adsorption of the second $\mathrm{Si}$ atom. The same applies to the gross features of the bonding between the first $\mathrm{Si}$ atom and the graphene sheet.

Table 1 Total energy and energy differences of the configurations under investigation (compare Fig. 1)

\begin{tabular}{llc}
\hline System & $E_{\text {total }}(\mathrm{Ry})$ & $\Delta E(\mathrm{eV})$ \\
\hline 1A & -1836.64 & 11.02 \\
1B & -1837.45 & \\
2A & -1848.56 & 2.45 \\
2B & -1848.74 & 0.54 \\
3A & -1859.91 & \\
3B & -1859.95 & \\
\hline
\end{tabular}


While so far we have obtained adsorption of two Si atoms at the defect in the graphene sheet, we still are not able to intercalate $\mathrm{Si}$ atoms. This changes when a third $\mathrm{Si}$ atom comes into play, see configurations $3 \mathrm{~A}$ and $3 \mathrm{~B}$. Comparison of the total energies of these two configurations shows that the latter is favorable by $0.54 \mathrm{eV}$, where an energy barrier of $1.39 \mathrm{eV}$ has to be overcome according to a nudged elastic band calculation. The value of the energy barrier is similar to $1.37 \mathrm{eV}$ obtained for diffusion of a monovacancy in graphene ${ }^{25}$ and is also in line with $2 \mathrm{eV}$ that is necessary for the transformation of a hexagon-hexagon pair into a pentagon-heptagon. ${ }^{26}$ We have also addressed the dependence of the energy barrier on the size of the defect by studying a divacancy. Because the Si atom here can penetrate the graphene sheet more easily, the energy barrier resulting from our nudged elastic band calculation is significantly reduced to $0.51 \mathrm{eV}$. The calculated $0.54 \mathrm{eV}$ energy gain by intercalation is the driving force of the intercalation process. It does hardly depend on the specific position of the intercalated Si atom between the defective graphene sheet and the $\mathrm{C}$ buffer layer. The basic mechanism for $\mathrm{Si}$ intercalation is thus migration through atomic defects in the graphene sheet. When the two stable adsorption sites at the defect are already occupied and a third $\mathrm{Si}$ atom approaches, the system can gain energy by shifting the first $\mathrm{Si}$ atom to an intercalation site and the second $\mathrm{Si}$ atom to the lower adsorption site. The third $\mathrm{Si}$ atom then occupies the upper adsorption site. During annealing, the deposited Si atoms by this mechanism pass one-by-one through the defect and form the intercalation layer.

Our results indicate that $\mathrm{Si}$ atoms prefer to form covalent $\mathrm{C}-\mathrm{Si}$ bonds rather than being intercalated. Therefore, the presence of defects is essential for $\mathrm{Si}$ intercalation. Since the $\mathrm{C}-\mathrm{Si}$ bonds are weaker than the $\mathrm{C}-\mathrm{C}$ bonds, the adsorption of $\mathrm{Si}$ cannot break the graphene network, which was found to be an important mechanism for $\mathrm{Li}$ intercalation. ${ }^{27} \mathrm{We}$ note that the buckling of the buffer layer is slightly stronger in the presence of the intercalated $\mathrm{Si}$ atom in configuration $3 \mathrm{~B}$ and that the interlayer spacing between the $\mathrm{C}$ buffer layer and the defective graphene sheet has increased to 4.2 $\AA$. Experimentally, $\mathrm{Si}$ intercalation is not possible at room temperature. The Si atoms stay on top of the defective graphene sheet. Our results show that adsorption of $\mathrm{Si}$ atoms at nondefective sites of the graphene sheet also comes along with energy gain, though much less than for adsorption at the defect. The first $\mathrm{Si}$ atom therefore will be attracted by the defect. However, additional energy is required for the jump to the lower adsorption site as well as to an intercalation site. As a consequence, intercalation is not possible at low temperature. Experimentally, the sample has to be annealed at $800{ }^{\circ} \mathrm{C}$ to enable intercalation. ${ }^{14}$

\section{Electronic structure}

We now turn to the electronic structures of the different configurations to study the influence of the intercalation. Band structures and Si partial densities of states (PDOSs) obtained for configurations 1A, 1B, 2B, and 3B are shown in Fig. 2. In the case of configuration 1A the Dirac cone is located $0.99 \mathrm{eV}$ below the Fermi energy $\left(E_{\mathrm{F}}\right)$, i.e., the system is strongly $n$-doped. The strong $n$-doping agrees with the experimental result, ${ }^{14}$ but the experimental shift of the Dirac cone is smaller, amounting to about $0.5 \mathrm{eV}$. The $\pi$ and $\pi^{*}$ bands (which form the Dirac cone) are purely contributed by the $\mathrm{C}$ atoms of the defective graphene sheet. The PDOSs show that the states of the isolated $\mathrm{Si}$ atom appear about $0.2 \mathrm{eV}$ above $E_{\mathrm{F}}$. Finally, the single band which
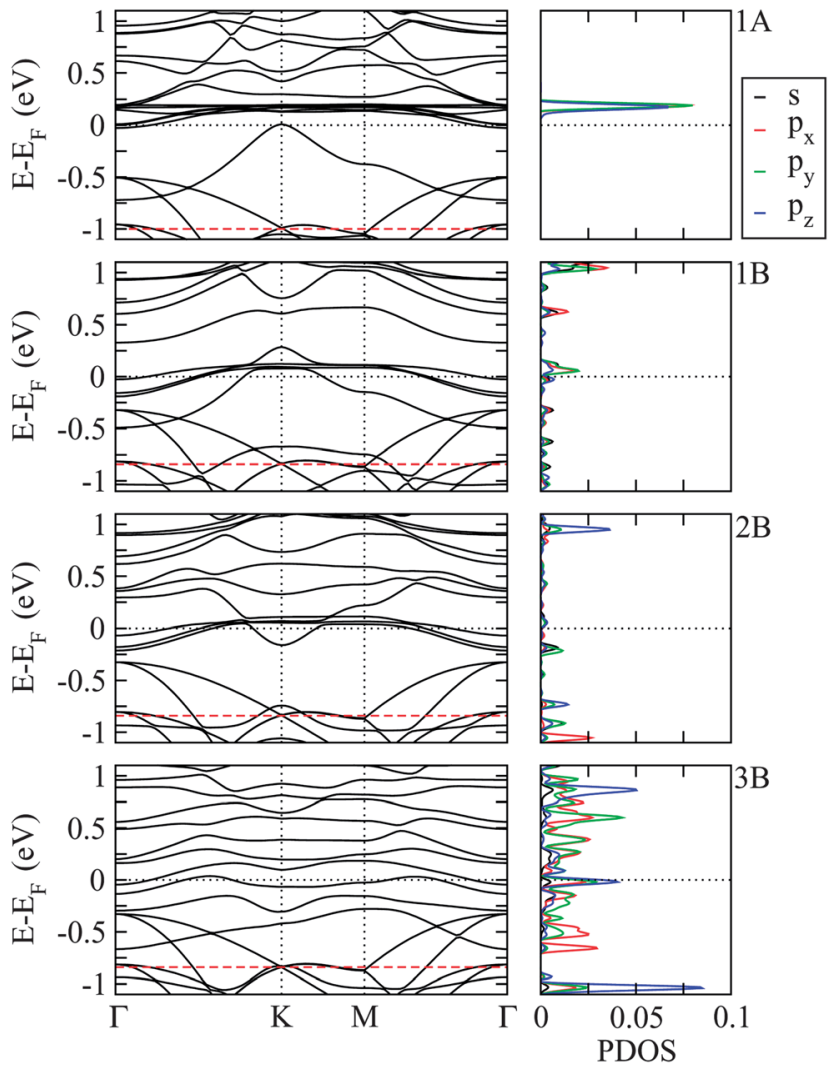

Fig. 2 Electronic band structures along the path $\Gamma-\mathrm{K}-\mathrm{M}-\Gamma$ with corresponding $\mathrm{Si} \mathrm{s}, \mathrm{p}_{\mathrm{x}}, \mathrm{p}_{\mathrm{y}}$, and $\mathrm{p}_{\mathrm{z}}$ PDOSs obtained for the configurations $1 \mathrm{~A}$, 1B, 2B, and 3B as defined in Fig. 1. Red dashed lines indicate the position of the Dirac cone. The PDOSs are averages over the Si atoms.

touches $E_{\mathrm{F}}$ along at the $\mathrm{K}$ point is a mixture of $\mathrm{p}_{\mathrm{z}}$ orbitals from the $\mathrm{C}$ buffer layer and the defective graphene sheet.

The $n$-type shift of the Dirac cone is reduced to $0.83 \mathrm{eV}$ in configuration $1 \mathrm{~B}$. The band originating from the $\mathrm{C} \mathrm{p}_{\mathrm{z}}$ orbitals now crosses $E_{\mathrm{F}}$. In contrast to configuration $1 \mathrm{~A}$, we find small $\mathrm{Si}$ contributions to the states just below and above $E_{\mathrm{F}}$. When the second $\mathrm{Si}$ atom is adsorbed, the situation is altered rather little. However, around the $\mathrm{K}$ point the nature of the states changes from hole-like to electron-like. Turning to configuration $3 \mathrm{~B}$, the Dirac cone is located $0.84 \mathrm{eV}$ below $E_{\mathrm{F}}$. We therefore find that the $n$-doping of the defective graphene sheet is not subjected to strong modifications during the intercalation process. After the intercalation, $\mathrm{Si}$ contributions to the electronic bands are present in a broad energy range around $E_{\mathrm{F}}$.

\section{Conclusion}

In conclusion, employing first principles calculations we have investigated the process of $\mathrm{Si}$ intercalation of graphene on a $\mathrm{SiC}(0001)$ substrate. In particular, we have established an intercalation mechanism which is based on $\mathrm{Si}$ migration through atomic defects of the graphene sheet. This mechanism has been described step by step in terms of total energy considerations. In addition, the effects of the intercalation on the electronic structure of the defective graphene have been analyzed, showing that the $n$-doping is hardly altered during the intercalation process. Unlike $\mathrm{H}, \mathrm{Li}, \mathrm{F}, \mathrm{K}$, and $\mathrm{Ge}$ intercalation, $\mathrm{Si}$ intercalation is found to require atomic defects in the graphene sheet. 


\section{References}

1 C. Berger, Z. Song, X. Li, X. Wu, N. Brown, C. Naud, D. Mayou, T. Li, J. Hass, A. N. Marchenkov, E. H. Conrad, P. N. First and W. A. de Heer, Science, 2006, 312, 1191.

2 K. V. Emtsev, A. Bostwick, K. Horn, J. Jobst, G. L. Kellogg, L. Ley, J. L. McChesney, T. Ohta, S. A. Reshanov, J. Röhrl, E. Rotenberg, A. K. Schmid, D. Waldmann, H. B. Weber and T. Seyller, Nat. Mater., 2009, 8, 203.

3 P. N. First, W. A. de Heer, T. Seyller, C. Berger, J. A. Stroscio and J.-S. Moon, MRS Bull., 2010, 35, 296.

4 C. Riedl, C. Coletti and U. Starke, J. Phys. D: Appl. Phys., 2010, 43, 374009.

5 K. V. Emtsev, F. Speck, T. Seyller, L. Ley and J. D. Riley, Phys. Rev. B: Condens. Matter Mater. Phys., 2008, 77, 155303.

6 C. Riedl, A. A. Zakharov and U. Starke, Appl. Phys. Lett., 2008, 93, 033106.

7 C. Riedl, C. Coletti, T. Iwasaki, A. A. Zakharov and U. Starke, Phys. Rev. Lett., 2009, 103, 246804.

8 S. L. Wong, H. Huang, Y. Wang, L. Cao, D. Qi, I. Santoso, W. Chen and A. T. S. Wee, ACS Nano, 2011, 5, 7662.

9 A. Varykhalov, J. Sánchez-Barriga, A. M. Shikin, C. Biswas, E. Vescovo, A. Rybkin, D. Marchenko and O. Rader, Phys. Rev. Lett., 2008, 101, 157601.

10 B. Premlal, M. Cranney, F. Vonau, D. Aubel, D. Casterman, M. M. D. Souza and L. Simon, Appl. Phys. Lett., 2009, 94, 263115.

11 T. P. Kaloni, M. Upadhyay Kahaly and U. Schwingenschlögl, $J$ Mater. Chem., 2011, 21, 18681.

12 Y. C. Cheng, T. P. Kaloni, G. S. Huang and U. Schwingenschlögl, Appl. Phys. Lett., 2011, 99, 053117.

13 J. Mao, L. Huang, Y. Pan, M. Gao, J. He, H. Zhou, H. Guo, Y. Tian, Q. Zou, L. Zhang, H. Zhang, Y. Wang, S. Du, X. Zhou, A. H. Castro Neto and H.-J. Gao, Appl. Phys. Lett., 2012, 100, 093101.

14 C. Xia, S. Watcharinyanon, A. A. Zakharov, R. Yakimova, L. Hultman, L. I. Johansson and C. Virojanadara, Phys. Rev. B. Condens. Matter Mater. Phys., 2012, 85, 045418.
15 F. Wang, K. Shepperd, J. Hicks, M. S. Nevius, H. Tinkey, A. Tejeda, A. Taleb-Ibrahimi, F. Bertran, P. Le Févre, D. B. Torrance, P. First, W. A. de Heer, A. A. Zakharov, and E. H. Conrad, arXiv:1111.2946.

16 Z.-H. Pan, J. Camacho, M. H. Upton, A. V. Fedorov, C. A. Howard, M. Ellerby and T. Valla, Phys. Rev. Lett., 2011, 106, 187002.

17 K. V. Emtsev, A. A. Zakharov, C. Coletti, S. Forti and U. Starke, Phys. Rev. B: Condens. Matter Mater. Phys., 2011, 84, 125423.

18 T. P. Kaloni, Y. C. Cheng, M. Upadhyay Kahaly and U. Schwingenschlögl, Chem. Phys. Lett., 2012, 534, 29.

19 T. P. Kaloni, M. Upadhyay Kahaly, Y. C. Cheng and U. Schwingenschlögl, Europhys. Lett., 2012, 98, 67003.

20 P. Giannozzi, S. Baroni, N. Bonini, M. Calandra, R. Car, C. Cavazzoni, D. Ceresoli, G. L. Chiarotti, M. Cococcioni, I. Dabo, A. Dal Corso, S. de Gironcoli, S. Fabris, G. Fratesi, R. Gebauer, U. Gerstmann, C. Gougoussis, A. Kokalj, M. Lazzeri, L. Martin-Samos, N. Marzari, F. Mauri, R. Mazzarello, S. Paolini, A. Pasquarello, L. Paulatto, C. Sbraccia, S. Scandolo, G. Sclauzero, A. P. Seitsonen, A. Smogunov, P. Umari and R. M. Wentzcovitch, J. Phys.: Condens. Matter, 2009, 21, 395502.

21 S. Grimme, J. Comput. Chem., 2006, 27, 1787.

22 T. P. Kaloni, Y. C. Cheng and U. Schwingenschlögl, J. Mater. Chem., 2012, 22, 919.

23 G. Henkelman, B. P. Uberuaga and H. Jonsson, J. Chem. Phys., 2000, 113, 9901.

24 Y. Hoshino, R. Fukuyama, Y. Matsubara, T. Nishimura, S. Tanaka, M. Kohyama and Y. Kido, Phys. Rev. B: Condens. Matter Mater. Phys., 2005, 71, 195331.

25 H. Zhang, M. Zhao, X. Yang, H. Xia, X. Liu and Y. Xia, Diamond Relat. Mater., 2012, 19, 1240.

26 Y. C. Cheng, H. T. Wang, Z. Y. Zhu, Y. H. Zhu, Y. Han, X. X. Zhang and U. Schwingenschlögl, Phys. Rev. B: Condens. Matter Mater. Phys., 2012, 85, 073406.

27 C. Virojanadara, A. A. Zakharov, S. Watcharinyanon, R. Yakimova and L. I. Johansson, New J. Phys., 2010, 12, 125015. 